Manard, M. \& Collette, F. (2014) - Influence du polymorphisme nucléotidique COMT sur la mémoire de travail et son vieillissement - Revue de Neuropsychologie (sous presse)

\title{
Influence du polymorphisme nucléotidique COMT sur la mémoire de travail et son
} vieillissement

Genetic influences on age-related cognitive changes: An illustration in working memory by means of the COMT single nucleotide polymorphism

\author{
Marine Manard ${ }^{1,2}$, Fabienne Collette ${ }^{1,2,3}$
}

${ }^{1}$ Centre de Recherche du Cyclotron, Université de Liège, Centre de Recherche du Cyclotron, Allée du 6 août $n^{\circ} 8$ (B30), 4000 Liège, Belgique.

${ }^{2}$ Département de Psychologie : Cognition et comportement, Université de Liège, Boulevard du Rectorat 3 (B33), 4000 Liège, Belgique.

${ }^{3}$ Fonds National de la Recherche Scientifique (FRS-FNRS), Belgique.

Adresse pour correspondance :

Fabienne Collette, Unité de Neuropsychologie, Boulevard du Rectorat 3 (B33), 4000 Liège, Belgique.

Tél: 0032436623 69, Fax: 0032436629 46, Email: f.collette@ulg.ac.be 
Manard, M. \& Collette, F. (2014) - Influence du polymorphisme nucléotidique COMT sur la mémoire de travail et son vieillissement - Revue de Neuropsychologie (sous presse)

\section{Résumé - Français (200 mots)}

L'objectif de cette revue est de synthétiser les connaissances sur l'influence du polymorphisme nucléotidique Catéchol-O-Méthyltransférase (COMT) val ${ }^{108 / 158}$ met sur la diminution des capacités de mémoire de travail (et plus particulièrement de ses aspects exécutifs) associée à l'avancée en âge. Par son implication dans les processus de dégradation de la dopamine, notamment au niveau préfrontal, ce polymorphisme semble avoir un rôle central dans l'efficacité de la mise en œuvre de processus exécutifs. En effet, plusieurs études suggèrent un avantage phénotypique de l'allèle met du polymorphisme COMT lors de tâches exécutives requérant une stabilité des représentations cognitives. Etant donné les modifications cérébrales observées avec l'âge au niveau frontal, le polymorphisme COMT semble constituer une piste pertinente pour comprendre les altérations cognitives liées à l'âge. En effet, suite à la diminution d'efficacité du système dopaminergique, les personnes âgées présentent des déficits de mémoire de travail plus ou moins importants selon leur génotype pour le polymorphisme COMT. De plus, l'activité cérébrale associée à la réalisation de ces tâches va également varier en fonction de ce polymorphisme. Ces résultats soulignent l'intérêt d'intégrer les approches de génétique comportementale et de neuroimagerie génétique afin d'approfondir notre compréhension du vieillissement cognitif.

Mots-clés: Fonctions exécutives ; Mémoire à court terme ; Dopamine ; Catéchol-OMéthyltransférase ; Vieillissement.

\section{Abstract - English (300 words)}

This review aims to synthetize the influence of the Catechol-O-Methyltransferase (COMT) $\mathrm{val}^{108 / 158}$ met single nucleotide polymorphism on the age-related working memory decline (and more particularly on the executive aspects). Involved in the catabolism of dopamine within the prefrontal cortex, the COMT polymorphism plays a central role in the efficiency of executive control in working memory. Indeed, several studies highlighted its influence in young adult populations, suggesting a phenotypic advantage of the Met allele of the COMT gene in executive tasks requiring stability of cognitive representations. As healthy aging was associated to a decline of dopamine availability in frontal areas, the COMT polymorphism could also be a relevant candidate to understand the age- 
Manard, M. \& Collette, F. (2014) - Influence du polymorphisme nucléotidique COMT sur la mémoire de travail et son vieillissement - Revue de Neuropsychologie (sous presse)

related cognitive decline in working memory / executive functions. As expected, a positive effect of Met polymorphism on these cognitive processes was observed in aging population. Moreover, cerebral and age-related compensatory activity related to working memory and executive tasks are also modulated by the COMT polymorphism, with a more efficient recruitment of brain areas in older carriers of the Met allele. These results clearly highlight the importance of behavioral genetic and neuroimaging genetic approaches to improve our understanding of cognitive and cerebral changes in normal aging, and more particularly to better comprehend the important heterogeneity of performance observed in that population.

Keywords: Executive function; Memory, Short-term; Dopamine; Catechol-O-Methyltransferase ; Aging. 
Manard, M. \& Collette, F. (2014) - Influence du polymorphisme nucléotidique COMT sur la mémoire de travail et son vieillissement - Revue de Neuropsychologie (sous presse)

Le déclin d'un certain nombre de processus cognitifs lors de l'avancée en âge a été associé à diverses modifications neurobiologiques, telles que la diminution des récepteurs dopaminergiques, l'atrophie corticale, ou encore des changements de l'activité cérébrale. Récemment, il a également été proposé que certains polymorphismes nucléotidiques pourraient influencer l'expression du déclin cognitif lors $\mathrm{du}$ vieillissement non-pathologique [voir par exemple: 1]. De par son implication dans la métabolisation de la dopamine ${ }^{1}$ au niveau frontal, l'enzyme Catéchol-O-Méthyltransférase (COMT) apparait particulièrement susceptible d'influencer les mécanismes cognitifs reliés au fonctionnement du cortex préfrontal, tels que la mémoire de travail (et plus particulièrement sa composante exécutive) en fonction du polymorphisme nucléotidique COMT val ${ }^{108 / 158}$ met $(\mathrm{rs} 4680)^{2}$ [2]. Cette revue s'intéresse donc aux effets que peut exercer le polymorphisme COMT sur la mémoire de travail et ses substrats cérébraux dans les populations jeunes et âgées, caucasiennes ${ }^{3}$ et non-pathologiques.

\section{Dopamine et mémoire de travail}

\subsection{Dopamine : aspects généraux}

En 1979, Brozoski, Brown, Rosvold, et Goldman ont observé qu'une réduction de concentration de dopamine et de noradrénaline dans le cortex préfrontal provoque une diminution drastique des compétences en mémoire de travail chez le singe. Répliqués chez l’humain, ces résultats suggèrent un rôle de première importance de la dopamine pour les mécanismes de mémoire de travail [voir par exemple; 5]. Produite au niveau du mésencéphale, la dopamine est distribuée à travers l'ensemble du cerveau par trois voies principales illustrées par la figure 1A [6]: la voie nigrostriée ; la voie mésolimbique et la voie mésocorticale. Les neurones dopaminergiques peuvent présenter deux niveaux d'activité distincts [7]: une activité tonique, correspondant à l'activité de base du système, soutenue et intrinsèquement régulée ; et une activité phasique, transitoire et déclenchée par la décharge des neurones dopaminergiques en fonction d'éléments extérieurs (détection d'un signal ou d'un évènement).

\footnotetext{
${ }^{1}$ La dopamine est un neurotransmetteur de la famille des catécholamines synthétisé au sein des neurones dopaminergiques.

${ }^{2}$ Selon la nomenclature, l'abréviation COMT (majuscule italique) réfère au gène et COMT (majuscule) est employée pour faire référence aux enzymes.

${ }^{3}$ La population caucasienne a été privilégiée au sein de cette revue étant donné les différences ethniques observées et ce particulièrement avec les populations asiatiques [voir 3].
} 
Manard, M. \& Collette, F. (2014) - Influence du polymorphisme nucléotidique COMT sur la mémoire de travail et son vieillissement - Revue de Neuropsychologie (sous presse)

\section{[Insérer Figure 1]}

Différents mécanismes pré et post-synaptiques (voir figure 1B) interviennent pour réguler la concentration de dopamine et sa fixation sur les récepteurs dopaminergiques [6]. Les neurones dopaminergiques transforment la tyrosine en dopa puis en dopamine, avant de l'acheminer vers la fente synaptique via des vésicules de stockage. Au moment où la dopamine est libérée dans la fente synaptique, plusieurs mécanismes interviennent, et notamment : 1) les transporteurs sélectifs DAT («dopamine active transporter »), en charge de la recapture d'une partie de la dopamine et de sa réintroduction dans le neurone, où elle sera soit stockée dans des vésicules, soit dégradée par l'enzyme mitochondriale mono-amine-oxydase (MAO) ; 2) L'enzyme COMT qui dégrade une partie de la dopamine au niveau de la fente synaptique; 3) enfin, une partie de la dopamine se fixe au niveau postsynaptique sur les récepteurs dopaminergiques des familles D1 et D2. Parmi ces différents mécanismes, deux apparaissent particulièrement importants de par leur action au niveau des régions préfrontales, et par conséquent leur influence sur les capacités de mémoire de travail : la capture de la dopamine par les récepteurs des familles D1 et D2 au niveau post-synaptique et sa dégradation dans la fente synaptique au moyen de l'enzyme COMT [8]. L'intérêt croissant des neurosciences pour l'enzyme Catéchol-O-Méthyltransférase (COMT), une enzyme ayant pour fonction d'O-méthyler toute substance présentant une structure catéchol, provient notamment de son rôle majeur dans la régulation des niveaux de dopamine, et ce particulièrement dans le cortex préfrontal, où elle en dégraderait plus de $60 \%$ [8].

Malgré certains débats, il semblerait que la relation existant entre la concentration de dopamine au niveau du cortex préfrontal et les performances de mémoire de travail suive une courbe en forme de $\mathrm{U}$ inversé [9]. Dès lors, des concentrations de dopamine trop basses entraineraient une diminution de performance mais des concentrations trop élevées seraient également délétères. De plus, le niveau optimum de dopamine dépendrait de la fonction cognitive exacte mise en jeu [9] (voir figure 2). Illustrant à la fois ce mécanisme et l'impact de facteurs environnementaux, Buckert et al. [10] ont observé que, suite à un protocole d'induction de stress aigu augmentant le taux de dopamine disponible, les individus val/val, ont obtenu de meilleures performances à la version 2-back d'une 
Manard, M. \& Collette, F. (2014) - Influence du polymorphisme nucléotidique COMT sur la mémoire de travail et son vieillissement - Revue de Neuropsychologie (sous presse)

tâche de mémoire de travail (niveau intermédiaire), par comparaison aux individus met/met, ceux-ci ayant dépassé leur niveau optimal de dopamine suite au protocole d'induction de stress.

\section{[Insérer Figure 2]}

\subsection{Dopamine et mémoire de travail : Modèles théoriques}

Plusieurs modèles fonctionnels ont été proposés afin de rendre compte du lien potentiel entre l'activité dopaminergique préfrontale et les performances cognitives. Ainsi, Cohen et Servan-Schreiber [11] ont proposé que la dopamine intervienne dans l'augmentation du ratio signal-bruit ${ }^{4}$, permettant à la fois le maintien des représentations contextuelles nécessaires à la bonne résolution de la tâche et un contrôle inhibiteur efficace de réponses prédominantes. Sur cette base, les modèles développés par la suite ont progressivement intégré différentes notions. Dreher et al. [12] ont modélisé l'influence des modes de transmission tonique et phasique des neurones dopaminergiques et la présence d'une courbe en forme de U inversé entre la réceptivité D1 et la performance cognitive [12]. Durstewitz et al. [13] tiennent compte de l'effet potentiellement opposé des récepteurs D1 et D2 [13]. Parmi ces différents modèles, les deux qui vont être présentés ci-dessous ont particulièrement intégré ces trois notions.

Cohen et al. [14] ont proposé que les modes antagonistes d'activité tonique et phasique puissent tous deux sous-tendre les représentations mnésiques au sein du cortex préfrontal. Cette relation antagoniste se marque notamment par le fait qu'une augmentation de la transmission tonique de dopamine semble inhiber sa transmission sur un mode phasique. Dans ce contexte, l'effet non-linéaire (et plus précisément en forme de $\mathrm{U}$ inversé) de la modulation dopaminergique sur la performance cognitive pourrait également s'expliquer par les effets de liaison différentielle de la dopamine aux récepteurs D1 et D2. La liaison sur les récepteurs D2, suite à une transmission phasique de dopamine, signalerait la présence d'une nouvelle information à intégrer en mémoire de travail, permettant aux réseaux préfrontaux de mettre à jour le système. La liaison aux récepteurs D1, suite à une transmission

\footnotetext{
${ }^{4}$ Plus précisément en augmentant la différentiation entre les niveaux de décharge de base des neurones et ceux provoqués par une stimulation externe.
} 
Manard, M. \& Collette, F. (2014) - Influence du polymorphisme nucléotidique COMT sur la mémoire de travail et son vieillissement - Revue de Neuropsychologie (sous presse)

tonique, permettrait quant à elle de limiter l'apparition de distraction dans le système par la stabilisation de l'activité corticale préfrontale jusqu'à l'obtention de la réponse désirée.

Dans le même ordre d'idées, Durstewitz et Seamans [15] ont proposé que l'impact, au niveau préfrontal, de la neuromodulation dopaminergique sur les performances de mémoire de travail s'opérerait selon deux modes de liaison distincts des récepteurs dopaminergiques: les états D1dominant («D1 state ») et D2-dominant («D2-state »), entrainant respectivement un maintien robuste ou un traitement flexible des représentations neuronales. La concentration de dopamine disponible à un moment donné va déterminer l'activité de ces deux classes de récepteurs. Plus précisément, de très hauts ou très bas niveaux de dopamine vont privilégier les effets associés à l'état D2-dominant tandis qu'une concentration intermédiaire de dopamine favorisera un état D1-dominant (voir Figure 3A). Cette proposition renvoie directement à l'observation d'une courbe en forme d'U-inversé sur laquelle Durstewitz et Seamans [15] suggèrent un impact du gène COMT. En effet, l'enzyme COMT influencerait les états D1-dominant et D2-dominant au sein des réseaux préfrontaux de par son rôle majeur de régulation de la concentration de dopamine.

\section{[Insérer Figure 3]}

\subsection{Dopamine et mémoire de travail : le rôle du polymorphisme COMT}

.Localisé sur le bras long du chromosome 22 (22q11.21), le gène COMT influence l'activité enzymatique permettant la dégradation extraneuronale de neurotransmetteurs tels que la dopamine, l'épinephrine et la norépinéphrine. Le gène COMT code pour deux formes d'enzymes largement distribuées : la forme «membrane-bound »(MB-COMT), préférentiellement exprimée dans les neurones cérébraux; et la forme «Soluble» (S-COMT) plutôt exprimée au sein de tissus périphériques, tels que le foie, le sang ou les reins. 
Manard, M. \& Collette, F. (2014) - Influence du polymorphisme nucléotidique COMT sur la mémoire de travail et son vieillissement - Revue de Neuropsychologie (sous presse)

Le polymorphisme nucléotidique $\mathrm{val}^{108 / 158}$ met du gène COMT $(\mathrm{rs} 4680)^{5}$, mis en évidence par Grossman et al. [16], implique la substitution d'un nucléotide de guanine pour un d'adénosine au niveau des codons 108 pour la forme soluble et 158 pour la forme «membrane-bound » provoquant la substitution d'un allèle de valine pour un allèle de méthionine dans la séquence d'acides aminés. Ce polymorphisme est dit «fonctionnel » étant donné qu'il entrainerait une différence de sensibilité thermique des enzymes suivant une distribution trimodale, avec une activité forte, intermédiaire, ou faible [17]. En effet, l'enzyme résultant de la variante met, moins stable à température corporelle, présente une activité enzymatique moindre. Ainsi, dans une étude post mortem, Chen et al. [17] ont observé, à $37^{\circ} \mathrm{C}$, une activité enzymatique $40 \%$ plus élevée des porteurs val par rapport aux porteurs met au sein du cortex préfrontal dorsolatéral. Enfin, de par la codominance ${ }^{6}$ des allèles de valine et de méthionine, les porteurs hétérozygotes val/met présentent une activité enzymatique intermédiaire.

\subsection{Impact du COMT en mémoire de travail : Aspects cognitifs et cérébraux}

Etant donné le lien entre le polymorphisme du gène COMT et la neuromodulation dopaminergique au niveau préfrontal, les recherches en neurosciences ont montré un intérêt croissant pour l'étude de l'impact du polymorphisme COMT sur le fonctionnement cognitif et plus particulièrement sur les processus de mémoire de travail et de contrôle exécutif. Plusieurs études mettent en évidence un avantage de l'allèle met, associé à une plus faible activité enzymatique, par rapport à l'allèle val lors de l'évaluation de ces processus [voir par exemple; 18]. Cet avantage des porteurs d'allèles met se traduit régulièrement, à performance égale, par une activité préfrontale moindre par rapport aux porteurs des allèles val, ce qui témoigne d'une plus grande efficacité des processus cérébraux [voir 19 pour une méta-analyse].

Cependant, d'autres études ont décrit une absence d'effet [20] voire un effet délétère de l'allèle met sur les performances exécutives et de mémoire de travail [voir 21 pour une méta-analyse]. Si ces

\footnotetext{
${ }^{5} \mathrm{La}$ fréquence allélique du polymorphisme COMT est relativement équilibrée au sein des populations caucasiennes et est estimée à $24.8 \%$ de met/met, $46 \%$ de val $/$ met et $29.2 \%$ de val/val (Hapmap CEU, $n=226$ individus)

${ }^{6}$ Le terme de codominance renvoie à la participation commune d'allèles dans l'expression phénotypique du gène, c'est-à-dire qu'ils présentent le même taux d'expression. Dès lors, les porteurs hétérozygotes auront une expression phénotypique intermédiaire aux variantes homozygotes.
} 
Manard, M. \& Collette, F. (2014) - Influence du polymorphisme nucléotidique COMT sur la mémoire de travail et son vieillissement - Revue de Neuropsychologie (sous presse)

résultats peuvent renvoyer à un manque de sensibilité des mesures comportementales, ils semblent également pouvoir s'interpréter en référence aux aspects tonique et phasique de neurotransmission dopaminergique.

En effet, selon Bilder et al. [22], la faible activité enzymatique associée à la présence de l'allèle met du gène COMT augmenterait l'activité tonique et diminuerait l'activité phasique au niveau sous-cortical. Cette faible activité serait également associée à des augmentations de concentration en dopamine au niveau cortical, entrainant l'augmentation de transmission au niveau des récepteurs D1 et la diminution au niveau des récepteurs D2 dans le cortex préfrontal (voir Figure 3B pour une présentation schématisée du mécanisme). Une forte activité de l'enzyme COMT (associée à la présence de l'allèle val) réduirait quant à elle la transmission tonique tout en augmentant l'activité phasique au niveau sous-cortical. Cette forte activité diminuerait les concentrations de dopamine corticale, entrainant une augmentation de transmission au niveau des récepteurs D2 avec une diminution au niveau des récepteurs D1. Au niveau comportemental, cette théorie propose que la présence de l'allèle met serait favorable à la stabilité des réseaux sous-tendant les représentations en mémoire de travail, mais rendrait la flexibilité ou la mise à jour des représentations actives plus laborieuse. A contrario, la présence de l'allèle val aurait pour conséquence une diminution de stabilité des réseaux neuronaux des représentations en mémoire de travail, rendant les capacités de flexibilité plus accessibles [voir par exemple; 23].

Complétant de manière intéressante la proposition de Bilder et al. [22], Durstewitz et Seamans [15] proposent que le polymorphisme COMT, de par son influence sur le niveau initial de dopamine intracérébrale, va directement moduler le mode de liaison des récepteurs dopaminergiques (les états D1dominant et D2-dominant; voir figure 3A). En effet, le niveau de concentration en dopamine initialement faible des individus homozygotes val/val entraine un régime de transmission favorisant les récepteurs D2, avantageant alors les processus de flexibilité cognitive. Les homozygotes met/met présenteraient quant à eux un régime tendant à favoriser les récepteurs D1, ce qui les avantage pour bon nombre de tâches de mémoire de travail nécessitant une stabilité des représentations. 
Manard, M. \& Collette, F. (2014) - Influence du polymorphisme nucléotidique COMT sur la mémoire de travail et son vieillissement - Revue de Neuropsychologie (sous presse)

Plusieurs études ont mis en évidence des résultats allant (au moins en partie) dans le sens de ces propositions. Au niveau comportemental, Rosa et al. [18] ont montré un avantage de l'allèle met lors de la réalisation d'une tâche de Stroop requérant de la stabilité cognitive, mais ne parviennent pas à mettre en évidence un effet des allèles val que ce soit pour la stabilité ou la flexibilité cognitive requise par la tâche ${ }^{7}$. A l'aide d'un paradigme de génération aléatoire de réponses motrices, Weiss et al. [24] ont observé que les individus met/met montrent plus de difficultés d'inhibition, se manifestant par la présence de stéréotypies, que les individus porteurs d'au moins un allèle val. De Frias et al. [25], ont montré qu'à performance comparable lors de la réalisation d'une tâche de mémoire de travail 2-back, il existe une activité transitoire du lobe temporal médian plus importante chez les porteurs des allèles met (reflétant une moindre efficacité de la composante de mise à jour de la tâche) mais une activité préfrontale soutenue plus importante pour les individus val/val que les individus met/met (reflétant une composante de maintien moins efficace). Enfin, Jaspar et al. [26], ont évalué l'activité soutenue et transitoire requise lors de l'administration d'un paradigme Stroop manipulant la proportion d'interférence afin de faciliter l'apparition d'un contrôle proactif (interférence fréquente entrainant une anticipation soutenue) ou réactif (interférence peu fréquente entrainant une activation transitoire au moment de son apparition) [voir 27]. Une activité plus importante au sein du cortex frontal moyen gauche a été observée chez les individus val/val, qui a été attribuée à une difficulté accrue à maintenir en mémoire les représentations du contexte de la tâche. Cette observation tend à soutenir l'hypothèse d'une transmission tonique plus faible chez ces individus, reflétant une moindre stabilité des processus cognitifs. Par ailleurs, dans cette étude, les individus porteurs des allèles met montrent également une activité diminuée dans l'operculum frontal en condition réactive, suggérant de meilleures compétences inhibitrices. Si ces derniers résultats ne permettent pas de conforter les prédictions concernant l'avantage de l'allèle val lorsque des processus de flexibilité entre différents processus cognitifs sont

\footnotetext{
${ }^{7}$ Dans cette étude, les auteurs [18] ont utilisé un paradigme Stroop variant le niveau de demande de la tâche en fonction de la présentation d'items congruents (mots « vert » ou " rouge » écrits dans la couleur correspondante) et non-congruents (mots « vert » ou « rouge » écrits dans la couleur opposée) ainsi qu'en proposant des parties de tâche constantes (répondre au mot vs. répondre à la couleur de l'encre) et des parties de tâche où les volontaires devaient alterner fréquemment entre les deux consignes.
} 
Manard, M. \& Collette, F. (2014) - Influence du polymorphisme nucléotidique COMT sur la mémoire de travail et son vieillissement - Revue de Neuropsychologie (sous presse)

requis $^{8}$, la moindre activité des homozygotes met au niveau de l'operculum frontal semble également renvoyer à leur meilleure stabilité des représentations cognitives, entrainant une meilleure gestion des situations de conflit.

Ces travaux indiquent donc, de façon générale, une influence du polymorphisme COMT sur les capacités de mémoire de travail et les régions préfrontales associées de sujets jeunes. Il semble plus particulièrement que la possession d'allèles met soit associée à une meilleure performance lorsque la tâche requiert une stabilité des représentations. L'influence de ce polymorphisme sur les compétences exécutives et de mémoire de travail lors du vieillissement normal sera examinée dans la suite de cette revue.

\section{Vieillissement non pathologique}

\subsection{Aspects neurobiologiques du vieillissement non pathologique}

\subsubsection{Déclin dopaminergique}

Plusieurs études post-mortem ont permis de mettre en évidence une diminution de la densité postsynaptique des récepteurs D1 et D2 avec l'avancée en âge. Le taux de déclin global de ces récepteurs peut être estimé à environ $10 \%$ par décennie [28]. Ces résultats ont été ultérieurement confortés par des travaux en imagerie cérébrale ayant observé une diminution de la densité des récepteurs D1 principalement au niveau frontal et une diminution de la quantité de récepteurs D2 tant au niveau frontal que dans les régions temporales, pariétales, occipitales ainsi que de l'hippocampe, l'amygdale et le thalamus [voir 28 pour revue]. Par ailleurs, Li et collaborateurs [29] ont simulé dans un modèle connexionniste [11] le déclin lié à l'âge de la modulation dopaminergique. Les résultats indiquent une diminution de réactivité neuronale entrainant une augmentation du bruit neuronal et rendant les patterns d'activation moins distinctifs, ce qui pourrait contribuer au déclin cognitif. Ce modèle réplique également la courbe en forme de U inversé reliant la performance cognitive à la concentration

\footnotetext{
${ }^{8}$ Bien que cette tâche n'ait pas été construite de façon à explorer spécifiquement les processus de flexibilité cognitive, on pourrait considérer que la condition réactive entraine, suite à la présentation d'un item interférent, une probabilité d'alternance plus importante entre le processus de lecture du mot et de dénomination de la couleur, étant donné la présence d'un grand nombre d'items facilitateurs induisant le processus de lecture.
} 
Manard, M. \& Collette, F. (2014) - Influence du polymorphisme nucléotidique COMT sur la mémoire de travail et son vieillissement - Revue de Neuropsychologie (sous presse)

de dopamine et suggère que l'avancée en âge s'accompagne d'un glissement sur la gauche de la courbe (Figure 4).

\section{[Insérer Figure 4]}

\subsubsection{Atrophie cérébrale}

Les modifications cérébrales survenant avec l'âge ont été étudiées via des recherches post-mortem mais également in vivo. Globalement, les études convergent vers la mise en évidence d'un déclin des structures cérébrales avec l'âge, touchant à la fois la matière grise et la matière blanche, et ce, particulièrement au niveau du cortex préfrontal [voir 30 pour revue]. [30]Selon certains auteurs, l'atrophie observée au sein de ces régions pourrait être influencée par le polymorphisme COMT [voir par exemple; 31]. Cependant l'étude de l'impact du polymorphisme COMT sur les aspects morphologiques du cerveau mène à des résultats inconsistants [31]. Par exemple, Cerasa et al. [31] ont observé, chez des adultes de 18 à 70 ans, que les porteurs d'allèles met présentent une épaisseur corticale supérieure à leurs homologues val, et ce dans des régions assez précises, telles que le sillon préfrontal inférieur et le sillon temporal supérieur. Taylor et al. [32], ont également observé des volumes hippocampiques et temporaux significativement moindres chez les individus homozygotes val par rapport aux porteurs d'allèles met. Par contre, d'autres études tendent à montrer des résultats opposés ou ne montrent simplement pas de lien entre le polymorphisme COMT et les caractéristiques morphologiques cérébrales d'individus sains [voir par exemple; 33]. Toutefois, si l'impact de ces modifications cérébrales structurelles sur le fonctionnement de la mémoire de travail a été observé à plusieurs reprises [voir 34 pour une présentation détaillée des travaux dans ce domaine], aucune étude ne s'est intéressée, à notre connaissance, à l'influence du polymorphisme COMT sur cette relation. Concernant la substance blanche, Papenberg et al. [35] ont mis en évidence dans un groupe de seniors que les porteurs homozygotes de l'allèle met montraient une meilleure intégrité de la substance blanche, ayant une fraction d'anisotropie plus élevée, particulièrement dans le cortex préfrontal, couplée à une plus faible diffusion moyenne que les porteurs d'allèles val. Cependant, ces auteurs n’ont pas mis en évidence d'effet génétique sur les capacités cognitives évaluées. Il semble important 
Manard, M. \& Collette, F. (2014) - Influence du polymorphisme nucléotidique COMT sur la mémoire de travail et son vieillissement - Revue de Neuropsychologie (sous presse)

de noter que ces auteurs n'ont pas inclus de tâche de mémoire de travail mais se sont intéressés à la vitesse de traitement, la mémoire épisodique, la mémoire sémantique, et les fluences verbales, expliquant peut-être cette absence d'effet.

\subsection{Vieillissement et mémoire de travail}

Avec l'âge, les compétences en mémoire de travail tendent à décliner, et ce notamment lorsque la quantité d'information à retenir est élevée, ou que son maintien nécessite une manipulation active du matériel [voir 36 pour revue]. Différentes pistes d'explication ont été proposées pour rendre compte de la survenue de ces difficultés, telles que la diminution des ressources de traitement disponibles, la diminution des capacités d'inhibition et de coordination de différents processus, un ralentissement de la vitesse de traitement de l'information, ou encore le déclin anatomique et fonctionnel des régions frontales.

De façon particulièrement intéressante, Braver et collaborateurs [37] ont proposé que l'altération du système dopaminergique puisse rendre compte des difficultés de mémoire de travail des personnes vieillissantes. En effet, les difficultés cognitives liées à l'âge dépendraient de la capacité à maintenir et à mettre à jour les représentations internes du contexte de la tâche ${ }^{9}$. La régulation de l'accès à cette information contextuelle, sa préservation de l'interférence et sa mise à jour, seraient influencées par les projections dopaminergiques vers le cortex frontal (et particulièrement les régions dorsolatérales).

Il est important de souligner que ce modèle offre un parallèle intéressant aux théories de neurotransmission tonique et phasique de la dopamine. En effet, Braver et Barch [37] postulent que ces deux modes de transmission pourraient intervenir de manière différentielle dans les processus de contrôle : le mode phasique favoriserait la mise à jour de l'information contextuelle au sein du cortex préfrontal suite à la présentation d'un stimulus saillant tandis que le mode tonique interviendrait dans le maintien actif des représentations contextuelles. Ces auteurs suggèrent de plus un déclin différentiel

\footnotetext{
${ }^{9}$ Ici définies comme toute information permettant de biaiser le traitement vers le circuit responsable de la bonne réalisation de la tâche (instructions, représentations du but de la tâche, stimulus particulier, ...). Dès lors, le contexte forme un ensemble de représentations au sein de la mémoire de travail qui vont orienter la manière d'utiliser les autres représentations, servant ainsi à la fois les fonctions mnésiques et de contrôle en mémoire de travail [37]
} 
Manard, M. \& Collette, F. (2014) - Influence du polymorphisme nucléotidique COMT sur la mémoire de travail et son vieillissement - Revue de Neuropsychologie (sous presse)

du système dopaminergique lors du vieillissement, avec dans un premier temps une altération des mécanismes de mise à jour uniquement (déclin du mode phasique) et ensuite une diminution des capacités de maintien (déclin du mode tonique) venant s'ajouter à celle des compétences de mise à jour.

\subsubsection{Aspects fonctionnels du vieillissement de la mémoire de travail}

De manière générale, les études d'imagerie fonctionnelle concernant le vieillissement non pathologique ont mis en évidence un lien entre diminution de performance et déclin d'activité dans certaines régions, résultat généralement interprété comme reflétant une difficulté d'engagement des réseaux requis pour la réalisation efficace de la tâche [voir par exemple; 38]. Cependant, la présence d'une activité plus importante dans certaines régions a également été observée chez les ainés. Cette sur-activation couplée à une performance cognitive moindre a été attribuée à un déclin des compétences d'activation sélective en fonction des processus requis par la tâche [voir par exemple; 39], à des processus de dédifférenciation [voir par exemple; 38], ou encore à des traitements cognitifs inefficaces [voir par exemple; 40]. Néanmoins, une performance similaire à celle de participants jeunes a également régulièrement été observée, suggérant que ces mécanismes de sur-activation pourraient refléter des mécanismes de compensation [41]. Dès lors, plusieurs modèles ont vu le jour afin d'expliquer des patterns récurrents d'activité dite «compensatoire » pour diverses fonctions cognitives sensibles à l'âge [voir 34 pour revue].

Ainsi, le modèle HAROLD [Hemispheric Asymetry Reduction in Older Adults; 42] propose que, pour obtenir une performance cognitive comparable à celle des sujets jeunes, les personnes âgées vont recruter des régions controlatérales supplémentaires, amenant à des patterns d'activité cérébrale plus symétriques. Le modèle PASA [Posterior-Anterior Shift with Aging; 43] propose de son côté que l'augmentation d'activité dans les régions antérieures (frontales) a pour fonction de compenser la diminution d'activité existant dans les régions postérieures. Le modèle CRUNCH [CompensationRelated Utilization of Neuronal Circuit Hypothesis; 44] propose quant à lui que le recrutement additionnel de ressources neuronales afin d'optimiser la performance n'est efficace que lorsque les 
Manard, M. \& Collette, F. (2014) - Influence du polymorphisme nucléotidique COMT sur la mémoire de travail et son vieillissement - Revue de Neuropsychologie (sous presse)

tâches ne sont pas trop exigeantes en ressources de traitement [44]. Le modèle STAC [Scaffolding Theory of Aging and Cognition; 45] semble offrir une approche intégrée de ces différents modèles. Il suggère qu'avec la dégradation liée à l'âge des circuits neuronaux, la réalisation de tâches cognitives devient plus complexe. Dès lors, des mécanismes adaptatifs prendraient place, et ce principalement au sein du cortex préfrontal, pour implémenter un circuit neuronal supplémentaire, complémentaire, voire alternatif, pour atteindre la performance visée. Enfin, Cabeza et Dennis [34] ont récemment proposé que les processus de compensation cérébrale mis en place lors du vieillissement ne concernent pas uniquement le recrutement de régions supplémentaires mais implique également une augmentation de la connectivité fonctionnelle au sein du cortex préfrontal.

Plusieurs de ces modèles (principalement les modèles HAROLD, STAC ainsi que le modèle proposé par Cabeza et Dennis [34]) évoquent l'impact potentiel de la neurotransmission dopaminergique sur la mise en place de processus de compensation. Dès lors, l'impact du polymorphisme COMT sur le vieillissement de la mémoire de travail et les modifications fonctionnelles observées avec l'âge semble d'un intérêt majeur dans la compréhension des mécanismes du vieillissement cérébral et cognitif.

\subsubsection{Influence du polymorphisme COMT val ${ }^{108 / 158}$ met sur le vieillissement de la mémoire de} travail

Alors que les études concernant l'impact du polymorphisme COMT sur le fonctionnement de la mémoire de travail et des fonctions exécutives ont amené à quelques données divergentes chez les jeunes adultes, les résultats obtenus chez les âgés indiquent, de façon assez consistante, un avantage des porteurs d'allèles met [voir par exemple; 46].

Sur base de ces résultats, Nagel et al. [1] ont proposé que l'âge puisse amplifier les effets du polymorphisme COMT sur la performance cognitive. En effet, lors de la réalisation d'une tâche exécutive (le Wisconsin Card Sorting Test), ces auteurs ont observé que les individus âgés commettent significativement plus d'erreurs persévératives que les participants jeunes. De plus, ils ont mis en évidence, chez les individus âgés, la présence d'un avantage significatif des homozygotes met par rapport à leurs homologues porteurs de deux allèles val. Le même type de résultat a été observé pour 
Manard, M. \& Collette, F. (2014) - Influence du polymorphisme nucléotidique COMT sur la mémoire de travail et son vieillissement - Revue de Neuropsychologie (sous presse)

une tâche de mémoire de travail spatiale comprenant entre 4 et 7 items. Sur cette base, les auteurs ont proposé que les individus âgés glisseraient naturellement vers la gauche de la courbe en forme de $\mathrm{U}$ inversé [voir 9 pour revue], les individus porteurs des allèles val manifestant, de par leur positionnement initial, un glissement plus important que les individus porteurs des allèles met (voir figure 4). Dans le même ordre d'idées, Bellander et al. [47] ont observé une performance significativement moindre en mémoire de travail chez les porteurs d'allèles val. Cependant, après un entrainement cognitif de 100 jours, ceux-ci ont présenté un gain plus important que les porteurs d'allèles met. Enfin, bien qu'une différence significative de performance apparait entre les jeunes adultes et les seniors, cette étude ne met pas en évidence d'interaction entre l'âge et le génotype, ne permettant pas de confirmer l'amplification de l'effet du polymorphisme COMT par l'âge.

Au niveau cérébral, Sambataro et al. [48] confortent les résultats de Nagel et al. [1] par l'observation d'une interaction entre le polymorphisme COMT val ${ }^{158}$ met d'une part, et la connectivité fonctionnelle entre le cortex préfrontal dorsolatéral et les autres régions du réseau préfronto-pariétal d'autre part, lors de la réalisation d'une tâche 1-back (sélectionnée afin d'obtenir une performance équivalente entre participants jeunes et âgés). Plus particulièrement, il apparait que, comparés aux jeunes adultes, les individus âgés homozygotes val présentent une connectivité fonctionnelle accrue entre le cortex préfrontal dorsolatéral et les autres régions du réseau préfronto-pariétal gauche. Ces auteurs suggèrent que le polymorphisme COMT et l'âge moduleraient l'activité et la connectivité fonctionnelle de régions cérébrales impliquées dans les processus de mémoire de travail, et proposent également que l'impact du polymorphisme COMT pourrait effectivement être amplifié par l'avancée en âge.

Dans le même ordre d'idées Nyberg et al. [49] ont observé que, lors d'une tâche de maintien d'informations verbales en mémoire à court terme, les adultes au-delà de 50 ans et les individus porteurs des allèles val (âgés quant à eux de 55 à 80 ans) recrutent de façon plus intensive le cortex préfrontal dorsolatéral pour atteindre une performance équivalente à celle de participants jeunes (de moins de 50 ans) ou d'individus âgés porteurs des allèles met. Rejoignant ainsi l'hypothèse de Cohen et al. [14], Nyberg et collaborateurs [49] proposent que les niveaux plus faibles de dopamine chez les adultes âgés et les porteurs des allèles val puissent altérer l'activation soutenue du cortex préfrontal 
Manard, M. \& Collette, F. (2014) - Influence du polymorphisme nucléotidique COMT sur la mémoire de travail et son vieillissement - Revue de Neuropsychologie (sous presse)

dorsolatéral, entrainant la nécessité d'un engagement plus important de ces régions déjà lors d'un simple maintien d'information. Par ailleurs, lorsqu'une manipulation alphabétique de l'information à retenir était requise, une activité plus importante est apparue au niveau du cortex préfrontal dorsolatéral chez les individus jeunes et ceux porteurs des allèles met uniquement. Ce dernier résultat suggère quant à lui que les individus âgés et ceux porteurs des allèles val ne parviennent pas à engager le cortex préfrontal dorsolatéral de façon optimale lorsque les processus cognitifs engagés par la tâche nécessitent une augmentation du niveau de dopamine. Dans leur ensemble, les résultats de cette étude confortent donc l'hypothèse d'un glissement vers la gauche sur la courbe en U inversé lors du vieillissement.

Finalement, il semblerait également que le polymorphisme COMT provoque une dédifférenciation plus ou moins importante des processus cognitifs avec l'avancée en âge. Papenberg et al. [50] ont en effet observé une corrélation latente entre une mesure de mémoire de travail spatiale et de mémoire épisodique verbale beaucoup plus importante chez les individus âgés homozygotes val par rapport à leurs homologues met, tandis que la taille de la corrélation ne diffère pas entre ces trois polymorphismes dans les groupes de sujets jeunes. Malheureusement, cette étude n'offre pas de données de neuroimagerie pour confirmer la présence d'un processus de dédifférentiation cérébrale.

\section{Conclusion}

Sur base des travaux présentés dans cette revue, un rôle du polymorphisme COMT sur le fonctionnement de la mémoire de travail et ses bases cérébrales semble actuellement bien établi. Des interrogations persistent toutefois sur la façon exacte dont s'exerce cette influence. De par l'existence d'une diminution du taux de dopamine disponible lors de l'avancée en âge, il est apparu pertinent de suspecter que les personnes vieillissantes présenteront des déficits de mémoire de travail plus ou moins importants selon leur génotype COMT. C'est effectivement ce que suggèrent les premiers travaux réalisés dans le domaine, et également que ce polymorphisme nucléotidique semble moduler l'activité cérébrale associée à la réalisation de ces tâches. 
Manard, M. \& Collette, F. (2014) - Influence du polymorphisme nucléotidique COMT sur la mémoire de travail et son vieillissement - Revue de Neuropsychologie (sous presse)

Si des études supplémentaires sont évidemment nécessaires pour conforter ces premiers résultats, il apparait toutefois que les approches de génétique comportementale et de neuroimagerie génétique sont indispensables à une compréhension approfondie du vieillissement cognitif. En effet, la prise en compte des aspects génétiques devrait permettre de mieux cerner les déterminants de l'importante hétérogénéité interindividuelle associée au vieillissement cognitif. Par exemple, nous pourrions supposer, en accord avec le modèle STAC [45] que certaines variations génétiques soient susceptibles, en l'absence de toute pathologie, de se répercuter sur la mise en place spontanée de stratégies de compensation cognitive.

Cette revue s'est centrée sur l'influence du polymorphisme COMT sur les processus de mémoire de travail dans le contexte du vieillissement normal. Cette démarche est évidemment valable pour certaines pathologies neurodégénératives telles que la maladie de Parkinson [voir par exemple; 51] ou Alzheimer [voir par exemple; 52] mais également pour d'autres gènes et processus cognitifs (par exemple le polymorphisme nucléotidique du $\mathrm{BDNF}^{10}$ et les capacités de mémoire épisodique). Il semble par ailleurs que certains gènes puissent être impliqués assez directement dans la constitution d'une réserve cognitive permettant de contrecarrer les effets du vieillissement normal et pathologique [53]. De plus, l'accumulation d'études longitudinales conjointement aux études transversales (chacune ayant ses avantages et inconvénients [voir par exemple; 54]) portant sur l'influence du polymorphisme COMT permettrait probablement d'éclairer le débat sur certains résultats non consensuels observés dans la littérature. L'analyse des relations entretenues par ces différents mécanismes (du niveau biochimique aux processus cognitifs de haut niveau) devrait donc permettre à terme d'augmenter drastiquement notre connaissance des processus liés au vieillissement, que ce dernier soit normal ou pathologique.

\section{$\underline{\text { Remerciements }}$}

Ce travail a été supporté par le Fonds National de la Recherche Scientifique F.R.S-FNRS (Fabienne Collette) et une Bourse Non-FRIA de l'Université de Liège (Marine Manard)

\footnotetext{
${ }^{10}$ Le facteur neurotrophique dérivé du cerveau (ou BDNF pour Brain-Derived Neurotrophic Factor) régule la croissance synaptique, la prolifération et la survie des neurones corticaux et module certains changements synaptiques tels que la potentialisation à long-terme.
} 
Manard, M. \& Collette, F. (2014) - Influence du polymorphisme nucléotidique COMT sur la mémoire de travail et son vieillissement - Revue de Neuropsychologie (sous presse)

\section{Légende des figures :}

Figure 1. (A) Projections dopaminergiques selon les trois voies principales: la voie nigro-striée (en rouge), partant de la substance noire vers le striatum; les voies mésolimbique et mésocorticale (en bleu), partant toutes deux de l'aire tegmentale ventrale et souvent groupées sous la dénomination de voie méso-cortico-limbique, projetant respectivement vers les régions limbiques (noyau accumbens, amygdale, hippocampe, cortex cingulaire antérieur) et le néocortex (cortex préfrontal orbitofrontal, dorsolatéral médian, cortex cingulaire). (B) Illustration de la synthèse et de la transmission dopaminergique entre deux neurones. Dans le neurone présynaptique, la dopamine (synthétisée à partir de la tyrosine transformée en L-Dopa), stockée dans les vésicules, est libérée dans la fente synaptique où trois voies de récupération/dégradation principales sont distinguées : 1) La dopamine va se lier aux récepteurs post-synaptiques des familles D1 et/ou D2; 2) Une certaine partie de dopamine sera recapturée dans le neurone présynaptique par les transporteurs sélectifs (DAT) afin d'être recyclée ou stockée en vue d'une utilisation ultérieure; 3) L'enzyme catéchol-O-méthyltransférase sera en charge de dégrader la dopamine restante dans la fente synaptique par O-méthylation.

Figure 2. La courbe en forme de $\mathrm{U}$ inversé [adaptée de 55] représentant la relation entre la concentration de dopamine cérébrale disponible et la performance cognitive. Cette relation suggère que de faibles comme de hauts niveaux de dopamine peuvent être délétères pour la performance cognitive. De plus, selon les exigences de la tâche proposée, certains auteurs postulent la présence de différents optimums, représentés ici par les tâches X (en jaune) et Y (en orange). La présence de ces différents optimums permet de rendre compte de l'effet positif d'un surdosage de dopamine pour certaines tâches.

Figure 3. (A) Représentation de la courbe en forme de U inversé concernant la relation entre l'activité des récepteurs $\mathrm{D} 1$ et $\mathrm{D} 2$ en fonction de la concentration de dopamine (les faibles et hauts niveaux entrainant un état dit «D2-dominant» et les niveaux intermédiaires favorisant un état «D1dominant » [15]. Les états D2-dominant sont associés à des caractéristiques favorisant les processus de flexibilité tandis que l'état D1-dominant semble favoriser le maintien des représentations nécessaires à 
Manard, M. \& Collette, F. (2014) - Influence du polymorphisme nucléotidique COMT sur la mémoire de travail et son vieillissement - Revue de Neuropsychologie (sous presse)

la réalisation d'une tâche cognitive. Les individus homozygotes val/val, présentant des niveaux de dopamine plus faibles au départ, se situent dès lors vers la gauche de la courbe, favorisant un état D2dominant. (B) Bilder et al. [22] suggèrent également un impact du polymorphisme COMT sur la neuromodulation dopaminergique. L'activité enzymatique plus importante des individus val/val provoquerait, au niveau sous-cortical, une diminution de la transmission tonique couplée d'une augmentation de la transmission phasique de dopamine, Etant donné la quantité plus faible de dopamine disponible au niveau cortical, la transmission aux récepteurs D2 sera favorisée au détriment des récepteurs D1. Ce mécanisme entraine, au niveau comportemental, une plus grande flexibilité accompagnée d'une moindre stabilité des représentations mentales. Les homozygotes met/met présentent le profil opposé à celui décrit ci - dessus pour leurs homologues val/val.

Figure 4. Représentation du glissement vers la gauche de la courbe des personnes âgées en fonction de leur génotype COMT. Illustration de la quantité de signal en fonction des périodes de vie et d'agents endogènes (ex: stress ou pathologie) ou exogènes (ex: prise de médicaments) [6]. 
Manard, M. \& Collette, F. (2014) - Influence du polymorphisme nucléotidique COMT sur la mémoire de travail et son vieillissement - Revue de Neuropsychologie (sous presse)

\section{Figures}

Figure 1

(A)

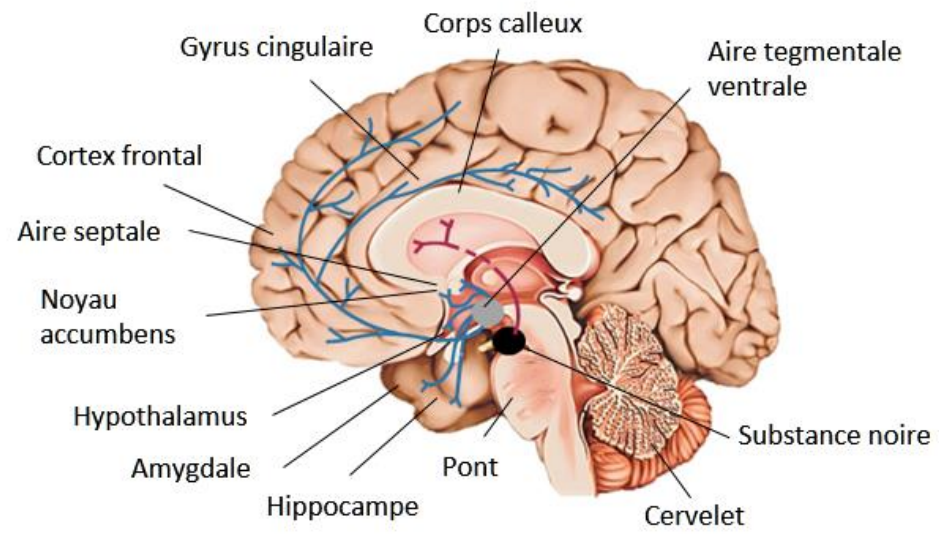

Figure 2

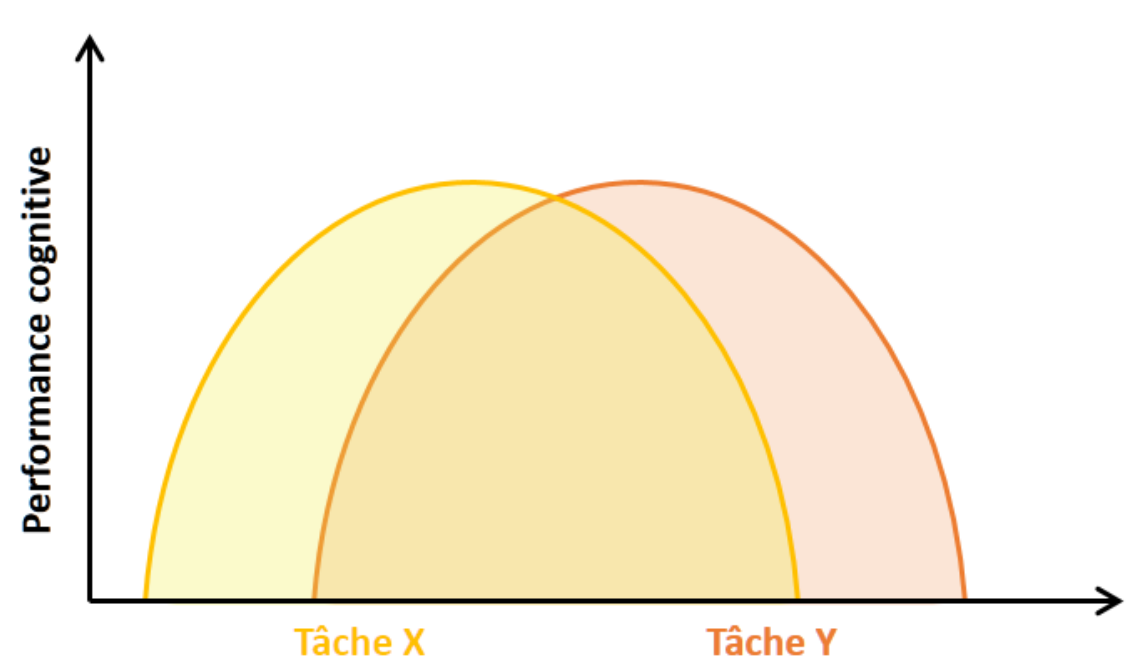

Concentration de dopamine

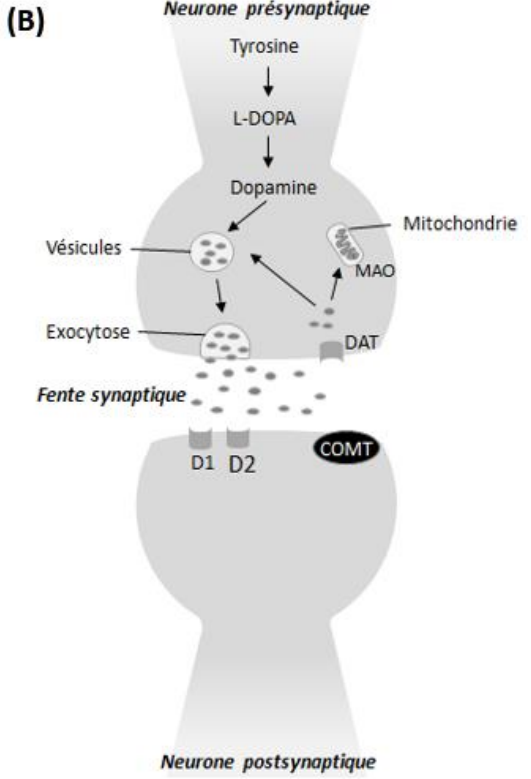

(B)

Neurone postsynaptique 
Manard, M. \& Collette, F. (2014) - Influence du polymorphisme nucléotidique COMT sur la mémoire de travail et son vieillissement - Revue de Neuropsychologie (sous presse)

Figure 3

(A)

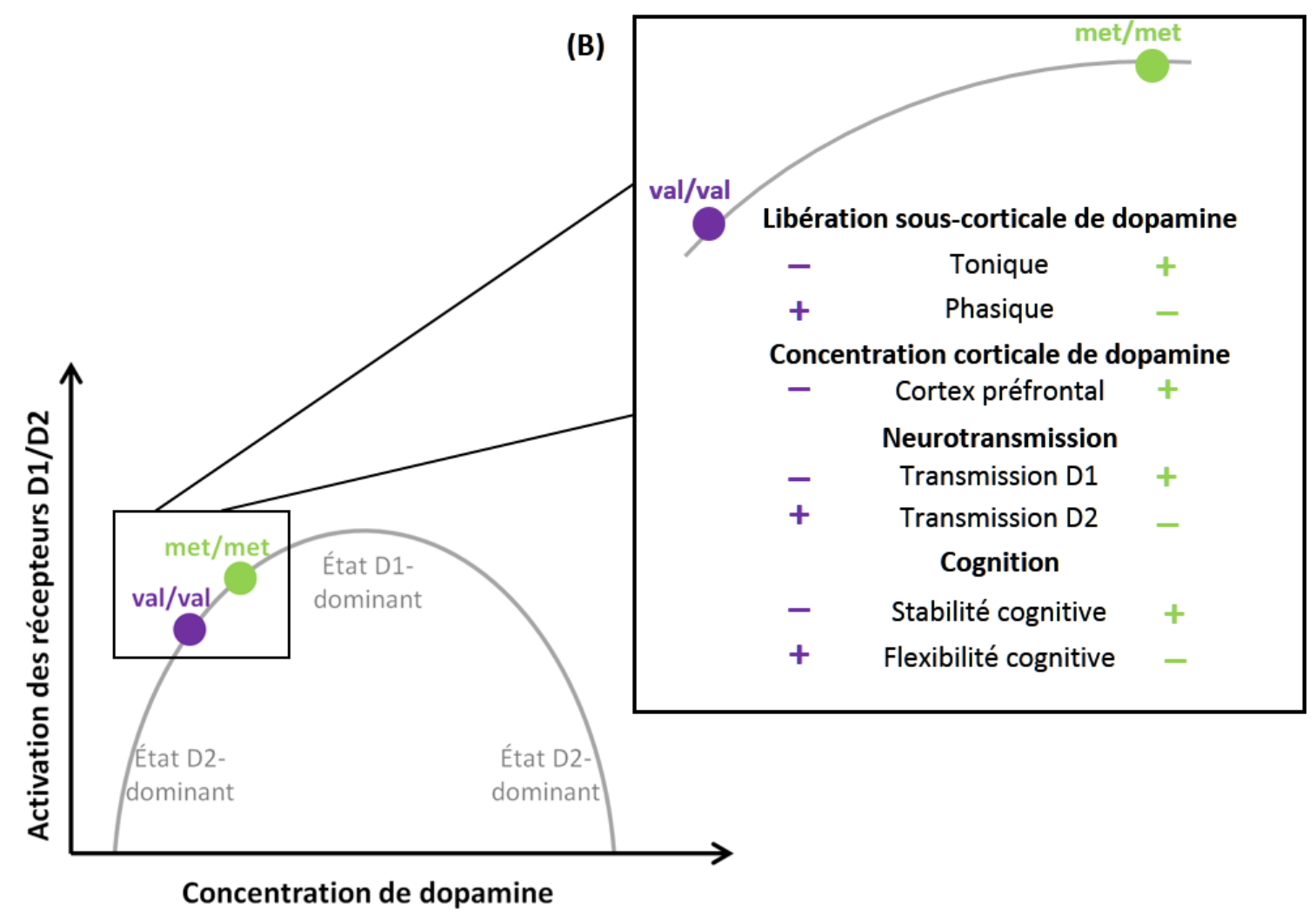

Figure 4

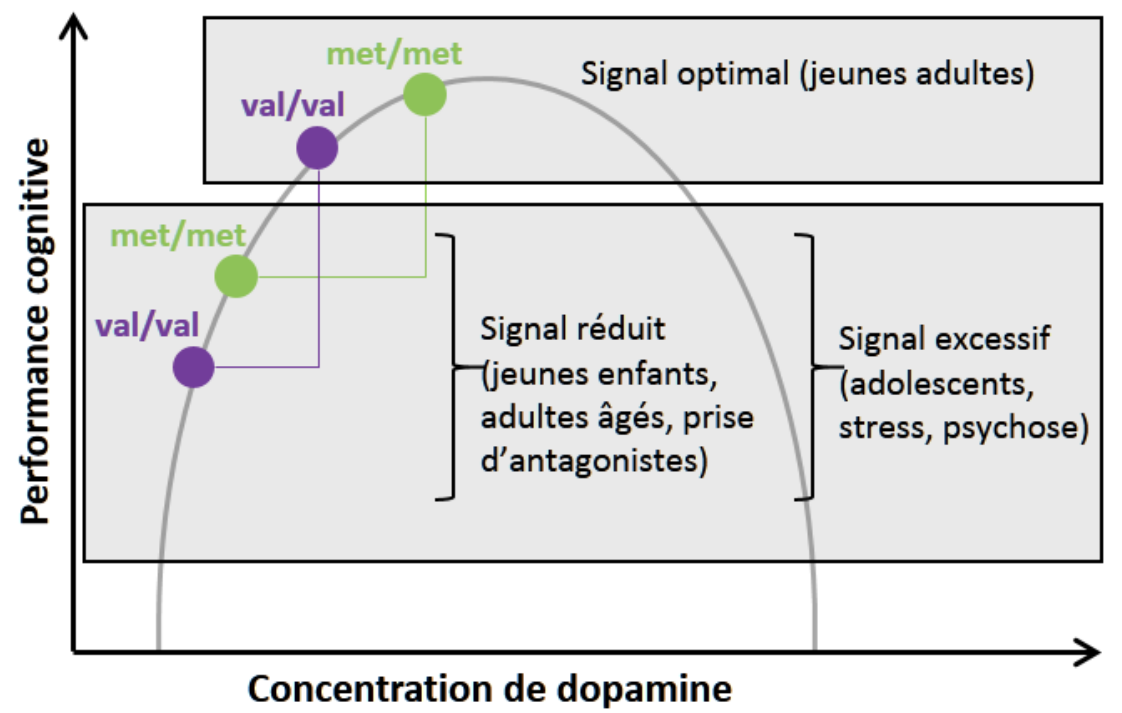


Manard, M. \& Collette, F. (2014) - Influence du polymorphisme nucléotidique COMT sur la mémoire de travail et son vieillissement - Revue de Neuropsychologie (sous presse)

\section{Références}

1. Nagel IE, Chicherio C, Li SC, von Oertzen T, Sander T, Villringer A et al. Human aging magnifies genetic effects on executive functioning and working memory. Frontiers in human neuroscience 2008; 2: 1-8.

2. Collette F, Van der Linden M, Laureys S, Delfiore G, Degueldre C, Luxen A et al. Exploring the unity and diversity of the neural substrates of executive functioning. Hum Brain Mapp 2005; 25(4): 409-23. 3. Wang Y, Li J, Chen C, Chen C, Zhu B, Moysis RK et al. COMT rs4680 Met is not always the 'smart allele': Val allele is associated with better working memory and larger hippocampal volume in healthy Chinese. Genes, brain, and behavior 2013; 12(3): 323-9.

4. Brozoski TJ, Brown RM, Rosvold HE, Goldman PS. Cognitive deficit caused by regional depletion of dopamine in prefrontal cortex of rhesus monkey. Science 1979; 205(4409): 929-32.

5. Liggins JTP. The roles of dopamine D1 and D2 receptors in working memory function. McGill Science Undergraduate Research Journal 2009; 4(1): 39-45.

6. Li SC, Lindenberger U, Bäckman L. Dopaminergic modulation of cognition across the life span. Neurosci Biobehav Rev 2010; 34(5): 625-30.

7. Grace AA. Phasic versus tonic dopamine release and the modulation of dopamine system responsivity: A hypothesis for the etiology of schizophrenia. Neuroscience 1991; 41(1): 1-24.

8. Karoum F, Chrapusta SJ, Brinjak R, Hitri A, Wyatt RJ. Regional effects of amphetamine, cocaine, nomifensine and GBR 12909 on the dynamics of dopamine release and metabolism in the rat brain. Br J Pharmacol 1994; 113: 1391-99.

9. Cools R, D'Esposito M. Inverted-U-shaped dopamine actions on human working memory and cognitive control. Biol Psychiatry 2011; 69(12): e113-25.

10. Buckert M, Kudielka BM, Reuter M, Fiebach CJ. The COMT Val158Met polymorphism modulates working memory performance under acute stress. Psychoneuroendocrinology 2012; : .

11. Cohen JD, Servan-Schreiber D. Context, cortex, and dopamine: A connexionnist approach to behavior and biology in shizophhrenia. Psychol Rev 1992; 99(1): 45-77.

12. Dreher JC, Burnod Y. An integrative theory of the phasic and tonic modes of dopamine modulation in the prefrontal cortex. Neural Networks 2002; 15: 583-602.

13. Durstewitz D, Seamans JK. The computational role of dopamine D1 receptors in working memory. Neural Networks 2002; 15: 561-72.

14. Cohen JD, Braver TS, Brown JW. Computational perspectives on dopamine function in prefrontal cortex. Curr Opin Neurobiol 2002; 12: 223-29.

15. Durstewitz D, Seamans JK. The dual-state theory of prefrontal cortex dopamine function with relevance to catechol-o-methyltransferase genotypes and schizophrenia. Biol Psychiatry 2008; 64(9): 739-49.

16. Grossman MH, Emanuel BS, Budarf ML. Chromosomal mapping of the human catechol-Omethyltransferase gene to 22q11.1----q11.2. Genomics 1992; 12(4): 822-25.

17. Chen J, Lipska BK, Halim N, Ma QD, Matsumoto M, Melhem S et al. Functional analysis of genetic variation in catechol-O-methyltransferase (COMT): Effects on mRNA, protein, and enzyme activity in post-mortem human brain. Am J Hum Genet 2004; 75: 807-21.

18. Rosa EC, Dickinson D, Apud J, Weinberger DR, Elvevag B. COMT Val158Met polymorphism, cognitive stability and cognitive flexibility: an experimental examination. Behavioral and brain functions : BBF 2010; 6: 53.

19. Mier D, Kirsch $P$, Meyer-Lindenberg A. Neural substrates of pleiotropic action of genetic variation in COMT: a meta-analysis. Mol Psychiatry 2010; 15(9): 918-27.

20. Wardle MC, de Wit H, Penton-Voak I, Lewis G, Munafo MR. Lack of association between COMT and working memory in a population-based cohort of healthy young adults. Neuropsychopharmacology 2013; 38(7): 1253-63.

21. Barnett JH, Scoriels L, Munafo MR. Meta-analysis of the cognitive effects of the catechol-Omethyltransferase gene Val158/108Met polymorphism. Biol Psychiatry 2008; 64(2): 137-44. 
Manard, M. \& Collette, F. (2014) - Influence du polymorphisme nucléotidique COMT sur la mémoire de travail et son vieillissement - Revue de Neuropsychologie (sous presse)

22. Bilder RM, Volavka J, Lachman HM, Grace AA. The catechol-O-methyltransferase polymorphism: relations to the tonic-phasic dopamine hypothesis and neuropsychiatric phenotypes. Neuropsychopharmacology 2004; 29(11): 1943-61.

23. Colzato LS, Waszak F, Nieuwenhuis S, Posthuma D, Hommel B. The flexible mind is associated with the catechol-O-methyltransferase (COMT) Val158Met polymorphism: evidence for a role of dopamine in the control of task-switching. Neuropsychologia 2010; 48(9): 2764-8.

24. Weiss EM, Schulter G, Fink A, Reiser EM, Mittenecker E, Niederstatter H et al. Influences of COMT and 5-HTTLPR Polymorphisms on Cognitive Flexibility in Healthy Women: Inhibition of Prepotent Responses and Memory Updating. PloS one 2014; 9(1): e85506.

25. De Frias CM, Marklund P, Eriksson E, Larsson A, Öman L, Annerbrink K et al. Influence of COMT gene polymorphism on $\mathrm{fMRI}$-assessed sustained and transient activity during a working memory task. J Cogn Neurosci 2009; 22(7): 1614-22.

26. Jaspar M, Genon S, Muto V, Meyer C, Manard M, Dideberg V et al. Modulating effect of COMT genotype on the brain regions underlying proactive control process during inhibition. Cortex 2013; : . 27. Braver TS. The variable nature of cognitive control: A dual mechanisms framework. Trends in cognitive sciences 2012; 16(2): 106-13.

28. Bäckman L, Nyberg L, Lindenberger U, Li SC, Farde L. The correlative triad among aging, dopamine, and cognition: current status and future prospects. Neurosci Biobehav Rev 2006; 30(6): 791-807.

29. Li SC, Lindenberger U, Sikström S. Aging cognition: From neuromodulation to representation. Trends in cognitive sciences 2001; 5(11): 479-86.

30. Raz N, Rodrigue KM. Differential aging of the brain: Patterns, cognitive correlates and modifiers. Neurosci Biobehav Rev 2006; 30: 730-48.

31. Cerasa A, Cherubini A, Quattrone A, Gioia MC, Tarantino P, Annesi G et al. Met158 variant of the catechol-O-methyltransferase genotype is associated with thicker cortex in adult brain. Neuroscience 2010; 167(3): 809-14.

32. Taylor WD, Züchner S, Payne ME, Messer DF, Doty TJ, MacFall JR et al. The COMT Val158Met polymorphism and temporal lobe morphometry in healthy adults. Psychiatry Res 2007; 155(2): 17377.

33. Rowe JB, Hughes L, Williams-Gray CH, Bishop S, Fallon S, Barker RA et al. The val158met COMT polymorphism's effect on atrophy in healthy aging and Parkinson's disease. Neurobiol Aging 2010; 31(6): 1064-8.

34. Cabeza R, Dennis NA. Frontal lobes and aging: Deterioration and compensation. In: Stuss DT, Knight RT, editors. Principles of frontal lobe function. 2 ed. New-York: Oxford University Press; 2013. 35. Papenberg G, Lovden M, Laukka EJ, Kalpouzos G, Keller L, Graff C et al. Magnified effects of the COMT gene on white-matter microstructure in very old age. Brain structure \& function 2014; : .

36. Braver TS, West RL. Working memory, executive control and aging. In: Craik FIM, Salthouse TA, editors. The Handbook of Aging and Cognition. 3 ed. New York: Psychology Press; 2008. p. 1-110.

37. Braver TS, Barch DM. A theory of cognitive control, aging cognition, and neuromodulation. Neurosci Biobehav Rev 2002; 26: 809-17.

38. Goh JO. Functional dedifferentiation and altered connectivity in older adults: Neural accounts of cognitive aging. Aging and Disease 2011; 2(1): 30-48.

39. Logan JM, Sanders AL, Snyder AZ, Morris JC, Buckner RL. Under-recruitment and nonselective recruitment: Dissociable neural mechanisms associated with aging. Neuron 2002; 33: 827-40.

40. Milham MP, Erickson KI, Banich MT, Kramer AF, Webb A, Wszalek T et al. Attentional Control in the Aging Brain: Insights from an fMRI Study of the Stroop Task. Brain Cogn 2002; 49(3): 277-96.

41. Reuter-Lorenz PA, Lustig C. Brain aging: reorganizing discoveries about the aging mind. Curr Opin Neurobiol 2005; 15(2): 245-51.

42. Cabeza R. Hemispheric Asymmetry Reduction in Older Adults: The HAROLD Model. Psychol Aging 2002; 17(1): 85-100.

43. Davis SW, Dennis NA, Daselaar SM, Fleck MS, Cabeza R. Que PASA? The posterior-anterior shift in aging. Cereb Cortex 2008; 18(5): 1201-9. 
Manard, M. \& Collette, F. (2014) - Influence du polymorphisme nucléotidique COMT sur la mémoire de travail et son vieillissement - Revue de Neuropsychologie (sous presse)

44. Reuter-Lorenz PA, Cappell KA. Neurocognitive aging and the compensation hypothesis. Association for Psychological Science 2008; 17(3): 177-82.

45. Park DC, Reuter-Lorenz P. The adaptive brain: aging and neurocognitive scaffolding. Annu Rev Psychol 2009; 60: 173-96.

46. Raz N, Dahle CL, Rodrigue KM, Kennedy KM, Land S. Effects of age, genes, and pulse pressure on executive functions in healthy adults. Neurobiol Aging 2011; 32(6): 1124-37.

47. Bellander M, Backman L, Liu T, Schjeide BM, Bertram L, Schmiedek F et al. Lower baseline performance but greater plasticity of working memory for carriers of the val allele of the comt val158met polymorphism. Neuropsychology 2014; : .

48. Sambataro F, Reed JD, Murty VP, Das S, Tan HY, Callicott JH et al. Catechol-O-methyltransferase valine(158)methionine polymorphism modulates brain networks underlying working memory across adulthood. Biol Psychiatry 2009; 66(6): 540-8.

49. Nyberg L, Andersson M, Kauppi K, Lundquist A, Persson J, Pudas S et al. Age-related and genetic modulation of frontal cortex efficiency. J Cogn Neurosci 2014; 26(4): 746-54.

50. Papenberg G, Backman L, Nagel IE, Nietfeld W, Schroder J, Bertram L et al. COMT Polymorphism and Memory Dedifferentiation in Old Age. Psychol Aging 2013; : 1-10.

51. Williams-Gray CH, Hampshire A, Barker RA, Owen AM. Attentional control in Parkinson's disease is dependent on COMT val 158 met genotype. Brain 2008; 131(Pt 2): 397-408.

52. Gennatas ED, Cholfin JA, Zhou J, Crawford RK, Sasaki DA, Karydas A et al. COMT Val158Met genotype influences neurodegeneration within dopamine-innervated brain structures. Neurology 2012; 78: 1663-69.

53. Robertson IH. A noradrenergic theory of cognitive reserve: implications for Alzheimer's disease. Neurobiol Aging 2013; 34(1): 298-308.

54. Fjell AM, Westlye LT, Grydeland H, Amlien I, Espeseth T, Reinvang I et al. Critical ages in the life course of the adult brain: nonlinear subcortical aging. Neurobiol Aging 2013; 34(10): 2239-47.

55. Cools R, Robbins TW. Chemistry of the adaptative mind. Philosophical Transactions of the Royal Society of London, Series A 2004; 362: 2871-88. 